\title{
MS34-05 | Analysing Aromatic Interactions: Clarity out of Complexity
}

Sovago, loana (Cambridge Crystallographic Data Centre, Cambridge, GBR); Wood, Peter (Cambridge Crystallographic Data Centre, Cambridge, AUT); McCabe, Patrick (Cambridge Crystallographic Data Centre, Cambridge, AUT); Pulido, Angeles (Cambridge Crystallographic Data Centre, Cambridge, GBR); Stevens, Joanna (Cambridge Crystallographic Data Centre, Cambridge, GBR)

The fundamental understanding of crystal structures and the structural drivers for stability is a key topic within chemical crystallography, as well being critical in an industrial context, particularly for the pharmaceutical and agrochemical industries. Here at the CCDC, we have been developing a suite of structure-based methods to help scientists better understand their crystal forms; to assess the stability and predict likely outcomes.

Currently, the community's solid form analysis methods focus mostly on analysing the quantity and quality of hydrogen bonds. This has been shown as a reasonably reliable indicator of solid form stability in systems where hydrogen bonding is present and dominant. There are, however, many solid forms where hydrogen bonds do not dominate, or are missing entirely. In these cases, the ability to analyse additional types of interactions is needed to rapidly provide a more holistic assessment of a crystal structure. Aromatic interactions are known to be another important interaction type for solid form stability and particularly so when hydrogen bonds are weaker, or not present.

We have developed a method to visualise and assess the strength of aromatic interactions in the context of a crystal structure, without needing to manually measure any geometric parameters. Using a sophisticated machinelearning approach, trained on high-level DFT-D3 energy calculations of benzene-benzene dimers, we can score phenyl ring-ring interactions quickly just from the observed geometry. The addition of effective assessment of phenyl-phenyl interactions to our solid form analysis toolbox takes us a big step closer towards more complete understanding of solid forms. 